Rountree, P. M. (1959). J. gen. Microbiol. 20, 620-633

\title{
Changes in the Phage-Typing Patterns of Staphylococci Following Lysogenization
}

\author{
By PHYLLIS M. ROUNTREE \\ Fairfax Institute of Pathology, Royal Prince Alfred Hospital, \\ Sydney, New South Wales, Australia
}

SUMMARY: Staphylococcal strains which showed a range of reactions with the phages $47 \mathrm{C}, 52,52 \mathrm{~A}, 80$ and 81 were examined for changes in phage sensitivity following artificial lysogenization. The lysogenic phages used were isolated from strains of three different phage-typing patterns and differed in serology. The changes in phage reactions thus produced included: (1) immunity to the $B$ phages, $52,52 \mathrm{~A}$ and 80 produced by a $B$ phage; (2) immunity to the $A$ phage 81 produced by a serologically unrelated $\mathrm{F}$ phage; (3) immunity to phage $47 \mathrm{C}$ and sensitivity to phages 52 and $52 \mathrm{~A}$ produced by a number of $\mathrm{A}$ phages. The mechanisms whereby a variety of typing patterns may be obtained within a related group of staphylococci are discussed.

In their study of the stability of phage-typing patterns in staphylococci, Williams \& Rippon (1952) used, among various sets of cultures, sequential ones from single sources such as nasal carriers, cultures from different sites on a single subject and cultures from different colonies from one primary culture plate. They found differences between members of these sets of cultures which indicated that some change in typing pattern occurred from day to day, both in vivo and in vitro. How much of this change was due to differences in the composition of the media used for typing and how much to changes in phage sensitivity of the staphylococci used was difficult to assess. However, their proposed rules for regarding strains of staphylococci as identical with or different from one another indicate that loss or gain of one 'strong reaction' may not be sufficient for regarding strains as different.

Smith (1948), Lowbury \& Hood (1953) and Rountree (1956) showed that changes in phage-typing pattern followed artificial lysogenization; these changes comprised losses in phage sensitivity and therefore indicated immunity in Lwoff's sense (1953). How frequent such changes may be in vivo is not known, but obviously they may have some importance in the interpretation of phage-typing results.

Following the identification in Australia of a new phage type of staphylococcus lysed only by a new phage 80 (Rountree \& Freeman, 1955) the origin of this new strain became of interest. Phage 80, a B phage serologically, was 'adapted' from phage $52 \mathrm{~A}$, of which it appears to be a virulent mutant since attempts to set up lysogenic systems with it have been unsuccessful. Strains of staphylococci lysed by both the B phages, 52 and $52 \mathrm{~A}$ are also lysed by phage 80 . Bynoe, Elder \& Comtois (1956) encountered in Canada a similar strain and identified it with a new phage $\mathbf{8 1}$, which is serologically $\mathbf{A}$ and also appears to 
be a virulent phage. The phage-typing pattern of the propagating strains of phages 80 and 81 is $80 / 81$.

In recent years, strains have been isolated in Australia which type as $80 / 81$, as 80 alone or as 81 alone. Strains typing as $52 / 52 \mathrm{~A} / 80 / 81$ are also found and may be fully or partially sensitive or resistant to phage $47 \mathrm{C}$. The staphylococcus responsible for the Bundaberg disaster (Kellaway, McCallum \& Tebbutt, 1928) has the phage pattern $52 / 52 \mathrm{~A} / 80 / 81$.

Since it was known that strains typing as 52/52 A (and also presumably sensitive to phages 80 and 81 ) had been isolated before any known occurrence of $80 / 81$ strains, the hypothesis that the $80 / 81$ pattern might have been derived from 52/52 A/80/81 by lysogenization with a phage giving immunity to phages 52 and $52 \mathrm{~A}$ was attractive. Preliminary investigations produced no evidence to support this hypothesis since no lysogenic B phages were found in $80 / 81$ strains. It was then decided to investigate the lysogenicity of a series of strains showing various combinations of reactions with the phages $47 \mathrm{C}, 52,52 \mathrm{~A}, 80$ and 81 and to transfer their lysogenic phages to other strains. If the phagetyping patterns within this series of strains depended on prophage immunity, then artificially lysogenized strains should resemble the strains used as sources of the prophages.

The strains selected for investigation on the basis of their typing patterns with the phages at routine test dilution were next examined with the undiluted phages. It then became apparent that they possessed characteristic patterns of 'inhibition' by high-titre phage filtrate. Williams \& Rippon (1952) drew attention to this 'inhibitory' effect of high titre phage filtrates and showed that the 'inhibitory' agent was no longer detectable in filtrates from which the phage had been removed by adsorption, by filtration or by neutralization with antisera. Unpublished observations in this laboratory indicate that the 'inhibition' is probably due to phage growth in populations of partly resistant staphylococci; it does not appear to be correlated with the prophage state of the cocci.

\section{METHODS}

Staphylococci. The strains of staphylococci used came from a variety of sources. The propagating strains of phages 80 and 81 are referred to as PS 80 and PS81 respectively. PS81 and phage 81 were obtained from the Staphylococcus Reference Laboratory, Colindale, London, England. Strain Bundaberg, referred to as $\mathbf{B}^{\prime} b$, was obtained from Dr J. Graydon of the Commonwealth Serum Laboratories, Victoria, Australia; in colonial appearance it corresponds to its original description, save for the loss of haemolysin. Other strains which will be referred to by number were selected from cultures sent from a number of places for routine phage typing. The phage-typing patterns and 'inhibition' patterns of representative strains are shown in Table 1. In addition to these strains, a number of others of similar patterns were used and will be mentioned in the text.

Preparation of phage stocks. The methods of preparing phage stocks on agar and of their titration have been described (Rountree, 1949a, 1952). Some 
phage stocks were also prepared by induction of lysogenic strains by ultraviolet irradiation (Gorrill \& Gray, 1956).

Lysogenicity. Strains of staphylococci were tested for lysogenicity by growing them in nutrient broth at $37^{\circ}$ for $2-3 \mathrm{hr}$., centrifuging and plating the supernatant fluids on appropriate indicator strains. PS80 was the indicator strain of choice for many of the lysogenic phages.

Table 1. Range of phage reactions in a series of staphylococci lysed by one or more of the phages $47 \mathrm{C}, 52,52 \mathrm{~A}, 80$ and 81

\begin{tabular}{|c|c|c|c|}
\hline Strain no. & Place of isolation & $\begin{array}{l}\text { Phage pattern } \\
\text { at RTD* }\end{array}$ & $\begin{array}{l}\text { pattern with } \\
\text { strong phage }\end{array}$ \\
\hline 8680 & Sydney & $47 \mathrm{C} / 52 / 52 \mathrm{~A} / 80 / 81$ & 一 \\
\hline 6338 & Coff's Harbour, N.S.W. & $47 \mathrm{C}+/ 52 / 52 \mathrm{~A} / 80 / 81$ & - \\
\hline 684 & Bairnsdale, Victoria & $52 / 52 \mathrm{~A} / 80 / 81$ & - \\
\hline Bundaberg & Bundaberg, Queensland & $52 / 52 \mathrm{~A} / 80 / 81$ & - \\
\hline PS 80 & Sydney & $80 / 81$ & $47 \mathrm{C} / 52 / 52 \mathrm{~A}$ \\
\hline PS81 & Canada & $80 / 81$ & $47 \mathrm{C} / 52 / 52 \mathrm{~A}$ \\
\hline 863 & Sydney & 80 & $47 \mathrm{C} / 52 / 52 \mathrm{~A}$ \\
\hline 864 & Sydney & 81 & $47 \mathrm{C} / 52 / 52 \mathrm{~A}$ \\
\hline
\end{tabular}

The antigenic character and host ranges of these phages after propagation on their indicator strains were determined by methods previously described (Rountree, 1949b, 1956). Determinations of host range were carried with the propagating strains of Wilson \& Atkinson (1945) and are, in general, expressed as ratios of number of plaques $/ \mathrm{ml}$. on the propagating strain of the particular phage stock to plaques $/ \mathrm{ml}$. on the indicator strains. Phages obtained from lysogenic strains are designated by the number or letter of their strain of origin followed by the number of the strain used for their propagation; e.g. phage $\mathrm{B}^{\prime} \mathbf{b} / 80$ is a lysogenic phage from strain Bundaberg grown in PS80.

Lysogenization. All phages used for lysogenization were filtered through Gradocol membranes of $0.8 \mu$ APD (average pore diameter) and tested for sterility before use. Strains to be lysogenized were grown in broth and then flooded on the surface of agar plates, dried and the undiluted phage spread on the surface of the plates. After incubation overnight at $37^{\circ}$, a small quantity of the secondary bacterial growth in the area of confluent phage lysis was streaked on to agar plates in such a way as to obtain separate colonies. Single non-eroded colonies from the edge of the inoculum were transferred to broth and incubated. No estimate has been made of the proportion of lysogenic responses obtained, but all the phages readily established lysogenic systems.

The lysogenized cultures were tested for the presence of lysogenic phage by plating their supernatant fluids on the homologous phage-sensitive strain; for contaminating lytic phage by flooding on agar and after incubation inspecting the resultant lawn for plaques; for resistance to the lysogenizing phage and for alterations in phage-typing pattern.

Lysogenized strains have their phage indicated in parentheses after the strain letter or number, e.g. PS80 $\left(B^{\prime} b\right)$ is lysogenized with a phage derived from strain Bundaberg. 


\section{RESULTS}

Alterations in phage sensitivity by lysogenization with phage 864

Staphylococcus 864 , lysed only by phage 81 , was 'inhibited' by phages $47 \mathrm{C}$, 52 and $52 \mathrm{~A}$ and gave no reaction with undiluted phage 80 ; that is, it was immune to phage 80 . It was lysogenic and its phage, 864, serologically $B$, could be propagated on strains sensitive to phage 80. A preparation of this phage made in strain 863 (phage 864/863) was used to lysogenize seven strains of various phage patterns. Table 2 records the changes in phage sensitivity that occurred. Six strains originally fully sensitive to phage 80 became immune. In the case of strain Bundaberg, the phage sensitivity was changed to 'inhibition'. Reactions with the related $\mathrm{B}$ phages, 52 and $52 \mathrm{~A}$, were either abolished or changed to 'inhibition'. Whether immunity or 'inhibition' followed lysogenization was not correlated with the original typing pattern

Table 2. Effect of lysogenization with phages 863 and 864 on phage-sensitivity patterns of staphylococci

\begin{tabular}{|c|c|c|c|c|c|}
\hline \multirow[b]{2}{*}{ Strain no. } & \multicolumn{5}{|c|}{ Phages } \\
\hline & $47 \mathrm{C}$ & 52 & $52 \mathrm{~A}$ & 80 & 81 \\
\hline \multicolumn{6}{|c|}{ Reactions before lysogenization } \\
\hline 863 & ( ) & ( ) & ( ) & $+t+t$ & - \\
\hline 864 & ( ) & ( ) & ( ) & - & ++++ \\
\hline PS 80 & () & ( ) & ( ) & ++++ & ++++ \\
\hline PS81 & ( ) & ( ) & ( ) & $++t+$ & ++++ \\
\hline 6351 & ( ) & ( ) & ( ) & ++++ & ++++ \\
\hline $\mathbf{B}^{\prime} \mathbf{b}$ & - & +++ & ++++ & $+t+t$ & $++t+$ \\
\hline 6338 & + & ++++ & $+t+t$ & ++++ & +++ \\
\hline 6922 & + & ++++ & $+++t$ & $+t+t$ & $+t+t$ \\
\hline 8680 & $++t+$ & +++ & $+t+t$ & $+t+t$ & ++++ \\
\hline
\end{tabular}

Reactions after lysogenization with phage 864

$\begin{array}{lccccc}\text { PS 80 } & (\text { ) } & - & - & - & ++ \\ \text { PS81 } & () & - & - & - & ++++ \\ \text { 6351 } & () & - & - & - & ++++ \\ \text { B'b } & - & () & () & () & () \\ \mathbf{6 3 3 8} & () & - & - & - & +++ \\ \mathbf{6 9 2 2} & ++ & () & () & - & ++++ \\ \mathbf{8 6 8 0} & () & - & - & - & ++++\end{array}$

Reactions after lysogenization with phage 863

$\begin{array}{llccccc}\text { PS80 } & & \text { () } & () & () & ++++ & - \\ \text { PS81 } & & \text { () } & ++++ & ++++ & ++++ & - \\ & \text { or } & \text { () } & () & () & ++++ & - \\ \mathbf{6 3 5 1} & & \text { () } & ++++ & ++++ & ++++ & - \\ & \text { or } & (\text { ) } & () & () & ++++ & - \\ \text { B'b } & & - & ++++ & ++++ & ++++ & - \\ \mathbf{6 3 3 8} & & (\text { ) } & ++++ & ++++ & ++++ & - \\ \mathbf{6 9 2 2} & & ++ & ++++ & ++++ & ++++ & - \\ \mathbf{8 6 8 0} & & () & ++++ & ++++ & ++++ & -\end{array}$

( ) = 'inhibition' with undiluted phage; $++++=$ confluent lysis with phage at routine test dilution (RTD); $++=100-200$ plaques with phage at RTD; $+=<100$ plaques with phage at RTD; $-=$ no reaction with undiluted phage. 
of the strain. After lysogenization there was no alteration in sensitivity to the A phage 81, with the exception of strain Bundaberg which showed 'inhibition'. Similarly, there was no immunity to the A phage $47 \mathrm{C}$, although reactions might be decreased in sensitivity.

The results showed that sensitivities to the $\mathrm{B}$ phages $52,52 \mathrm{~A}$ and 80 were closely linked and that the introduction of a $\mathrm{B}$ prophage resulted in partial or complete resistance to all three phages. When the typing patterns at the routine test dilutions only of phages $52,52 \mathrm{~A}$ and 80 are taken into account, all the lysogenized strains and strain 864 are identical. Phage 864/863 therefore in its prophage state blocked the multiplication of phage 80 in previously sensitive cocci; it is not, however, identical with phage 80 . It behaved as a temperate and not as a virulent phage and its host range (Table 3) differed in several respects from that of our stock of phage 80 . In particular, it did not lyse propagating strains $31 / 44,42 \mathrm{C}$ or 52 , and it produced 100 times fewer plaques on propagating strain $52 \mathrm{~A}$. It was concluded that the presence of this prophage was responsible for immunity to phage 80 in strain 864 .

Table 3. Host ranges of phage 80 and phage $864 / 863$

$\begin{array}{crc}\text { Phage titre/ml.: } & \begin{array}{c}\text { Phage } 80 \\ 9 \times 10^{8} \\ \text { Ratio: titre on propagating strain to } \\ \text { titre on indicator strain } \\ \text { strain }\end{array} & \begin{array}{c}\text { Phage 864/863 } \\ 2.5 \times 10^{8}\end{array} \\ 6 & - & 1,000 \\ 31 / 44 & 1,000 & - \\ 42 \mathrm{C} & 10,000 & - \\ 44 \mathrm{~A} & 1,000 & 1,000 \\ 47 & 500 & 200 \\ 47 \mathrm{C} & 1,000 & 200 \\ 52 & 100 & - \\ 52 \mathrm{~A} & 150 & 10,000 \\ 80 & 1 & 1\end{array}$

Alterations in phage sensitivity by lysogenization with phage 863

Staphylococcus 863 was lysed only by phage 80 , it was 'inhibited' by undiluted phages $47 \mathrm{C}, 52$ and $52 \mathrm{~A}$ and was immune to phage 81 . It was lysogenic, and its phage 863 could be propagated in strains lysed by phage 81 . When phage $863 / 864$ was prepared it proved to be serologically $F$. The same strains used for lysogenization with phage 864 were lysogenized with phage 863/864 and the resulting changes in phage sensitivity are shown in Table 2. Lysogenization with this $\mathrm{F}$ phage resulted in immunity to phage 81 in all seven strains. There was little or no effect on reactions with phage $47 \mathrm{C}$. In general, there was little effect on reactions with the $\mathbf{B}$ phages, but two anomalous cultures PS81 (863) and 6351 (863) were isolated which will be discussed in a later section of this paper.

It was concluded that the presence of the $\mathbf{F}$ phage in the prophage state in cocci of strain 863 was responsible for the immunity to the A phage 81 . Since this was a surprising result, the stocks of phage 81 were confirmed as being 
serologically $\mathbf{A}$. There was no crossing in neutralization tests with anti-A and anti-F sera (Rountree, $1949 b$ ). Strain 863 therefore provides an exception to previous findings that there is no cross-immunity between phages of unrelated antigenic structure. It should be noted, however, that the host ranges of phages 81 and $863 / 864$ are very similar, the main differences being lack of minor reactions by phage 863 on the propagating strains 7 and $42 \mathrm{C}$.

\section{The effect of lysogenization with $A$ phages on $80 / 81$ strains}

In two cultures, PS 81 (863) and 6351 (863) shown in Table 2, lysogenization with the $F$ phage 863 had apparently resulted in a change of sensitivity to phages 52 and $52 \mathrm{~A}$. Instead of being 'inhibited' by high-titre phages, the lysogenized cocci were as sensitive as the propagating strains of phages 52 and 52 A. This was not however a constant feature of the lysogenized clones of these two strains since others still showed 52/52 A 'inhibition'.

Table 4. Effect of lysogenization with $\mathrm{A}$ phages, $\mathrm{B}^{\prime} \mathrm{b}$ and 684 , on the phage typing patterns of $80 / 81$ strains

\begin{tabular}{|c|c|c|c|c|c|}
\hline \multirow{3}{*}{ Strain no. } & \multicolumn{5}{|c|}{ Phages } \\
\hline & $47 \mathrm{C}$ & 52 & $52 \mathrm{~A}$ & 80 & 81 \\
\hline & & & Reaction & & \\
\hline $\begin{array}{c}\text { PS 80, PS81, 6351, } \\
6352,6402,9183\end{array}$ & () & () & () & ++++ & ++++ \\
\hline PS $80\left(B^{\prime} b\right)$ & - & ++++ & +++ & ++++ & ++++ \\
\hline PS 80 (684) & - & ++++ & ++++ & ++++ & ++++ \\
\hline PS81 ( $\left(B^{\prime} b\right)$ & - & ++++ & ++++ & ++++ & ++++ \\
\hline PS81 (684) & - & ++++ & ++++ & ++++ & ++++ \\
\hline $6351\left(\mathrm{~B}^{\prime} \mathrm{b}\right)$ & - & ++++ & ++++ & ++++ & ++++ \\
\hline $6351(684)$ & - & ++++ & ++++ & ++++ & ++++ \\
\hline $6352\left(\mathrm{~B}^{\prime} \mathrm{b}\right)$ & - & ++ & ++ & ++ & ++ \\
\hline $6352(684)$ & - & ++ & ++ & ++ & ++ \\
\hline $6402\left(\mathrm{~B}^{\prime} \mathrm{b}\right)$ & - & ++++ & $+++t$ & ++++ & $+t$ \\
\hline $6402(684)$ & - & ++++ & ++++ & ++++ & ++++ \\
\hline $9183\left(B^{\prime} b\right)$ & - & $+++t$ & ++++ & ++++ & ++++ \\
\hline $9183(684)$ & - & ++++ & $+++t$ & ++++ & ++++ \\
\hline
\end{tabular}

Dr Joan Rippon, in a personal communication, stated that the transfer of certain A prophages to strains typing as $\mathbf{8 0 / 8 1}$ resulted in strains which typed as 52/52 A/80/81. A search for similar lysogenic phages was made among our strains and Dr Rippon's observations confirmed. Two strains, Bundaberg and 684, which type as 52/52 A/80/81 were found to be lysogenic for A phages to which strains typing as $80 / 81$ are fully sensitive. These two phages, $B^{\prime} b$ and 684, were propagated in PS80 and used to lysogenize six strains typing as $80 / 81$. All the lysogenized clones typed as $52 / 52 \mathrm{~A} / 80 / 81$; in addition, their 'inhibition' with phage $47 \mathrm{C}$ disappeared and they became immune to this phage (Table 4). The presence or absence of reactions with phage $47 \mathrm{C}$ was therefore used as an index of the presence or absence in strains typing as $52 / 52 \mathrm{~A} / 80 / 81$ of an A prophage capable of 'converting' 80/81 strains to $52 / 52 \mathrm{~A} / 80 / 81$. The phages which produce this change in phage sensitivity will 
be referred to as 'converting' phages and the change termed 'conversion' without implying the exact mechanism involved. Five further strains typing as $52 / 52 \mathrm{~A} / 80 / 81$ were found to be lysogenic for these 'converting' phages while eight strains typing as 52/52 A/80/81 but showing 'inhibition' with undiluted phage $47 \mathrm{C}$ were devoid of lysogenic activity when they were plated on PS 80.

\section{Characters of the 'converting' phages}

Five of the 'converting' phages, namely, $\mathrm{B}^{\prime} \mathrm{b}, 684,303,313$ and 315 , were propagated in PS80 and their host ranges and serology determined. The phages were serologically A. Their host ranges (Table 5) show that the first

Table 5. Host ranges of 'converting' phages of serological group A

\begin{tabular}{|c|c|c|c|c|c|c|}
\hline & \multicolumn{5}{|c|}{ Phage } \\
\hline & & $\mathbf{B}^{\prime} \mathbf{b}$ & 684 & 308 & 313 & 315 \\
\hline \multicolumn{2}{|c|}{$\begin{array}{l}\text { Titre/ml. on propagating } \\
\text { strain PS } 80\end{array}$} & $6.5 \times 10^{7}$ & $1.6 \times 10^{9}$ & $1.2 \times 10^{10}$ & $3 \cdot 3 \times 10^{9}$ & $6 \times 10^{8}$ \\
\hline & & \multicolumn{5}{|c|}{ Ratio: titre on PS80 to titre on indicator strain } \\
\hline \multirow{7}{*}{ Indicator strain } & 6 & 10 & 10 & 16 & 18 & 20 \\
\hline & $31 / 44$ & 20 & 10 & 20 & 30 & 30 \\
\hline & $31 \mathrm{~A}$ & - & 40,000 & 30,000 & 30,000 & $1 \cdot 5$ \\
\hline & $42 \mathrm{C}$ & - & 一 & - & - & 300 \\
\hline & $42 \mathrm{E}$ & 6,500 & 300 & $\mathbf{5 , 0 0 0}$ & 5,000 & 200 \\
\hline & 47 & - & 300,000 & 10,000 & 10,000 & 100,000 \\
\hline & $47 \mathrm{C}$ & 3 & 8 & 6 & 10 & 10 \\
\hline
\end{tabular}

four phages resemble each other closely while phage 315/80 differs in its ability to attack strain $31 \mathrm{~A}$ and, to a less extent, strain $42 \mathrm{C}$. The host ranges of these phages differ from that of the A phage, 47C (Rountree, 1956). All five phages were easy to propagate and produced clear plaques on PS80, those of phage 684/80 being smaller than those of the other four. When they were applied undiluted to PS80, secondary resistant bacterial growth appeared in the areas of confluent lysis and was found to contain lysogenized cocci.

The original lysogenic hosts of these phages and cultures of PS 80 lysogenized with each phage were tested for cross-immunity to the phages. In these tests, the phages were applied undiluted and the results (Table 6) indicated reciprocal cross-immunity and hence identity of phages $B^{\prime} b$ and 308 . Undiluted phage 684 produced 'inhibition' on all strains save its homologous strain and strain Bundaberg; these areas of inhibition in most cases contained scattered plaques. Similar appearances were observed with phage 313, which also gave 'inhibition' with its homologous strain 313 although not with strain Bundaberg. These reactions were considered to indicate that immunity to phages 684 and 313 was not absolute and also that the phage preparations probably contained mutant particles which were the cause of the scattered plaques. When the four phages were tested at higher dilutions, these reactions were not detectable and the phages would be regarded as identical. Phage 
$315 / 80$ was clearly different from the other four phages. Strains 315 and PS 80 (315) were identical and immune to all five phages, save for the minor reactions with phages 684 and 313 . Nevertheless, all the other strains were fully sensitive to phage 315, thus providing an example of non-reciprocity in prophage immunity. Preliminary observations indicate that these phages are readily inducible by ultra-violet light.

Table 6. Cross-immunity between 'converting' phages

\begin{tabular}{|c|c|c|c|c|c|}
\hline \multirow{3}{*}{ Strain } & \multicolumn{5}{|c|}{ Phage } \\
\hline & $\mathrm{B}^{\prime} \mathrm{b} / \mathbf{8 0}$ & $684 / 80$ & $308 / 80$ & $313 / 80$ & $315 / 80$ \\
\hline & \multicolumn{5}{|c|}{ Lytic reactions with undiluted phages } \\
\hline $\mathbf{B}^{\prime} \mathbf{b}$ & - & - & - & - & $(++++)$ \\
\hline 684 & - & - & - & () & $(++++)$ \\
\hline 308 & - & $(++)$ & - & $(+++)$ & ++++ \\
\hline 313 & - & $(++)$ & - & ( ) & $(++++)$ \\
\hline 315 & - & () & - & $+t$ & - \\
\hline PS $80\left(B^{\prime} b\right)$ & - & $(++)$ & - & $(++)$ & $++t$ \\
\hline PS 80 (684) & - & $(++)$ & - & $(++)$ & $(+t+t)$ \\
\hline PS 80 (308) & - & ++ & - & $(+++)$ & $(++++)$ \\
\hline PS80 (313) & - & $(+t)$ & - & $(++)$ & $(t+t+)$ \\
\hline PS80 (315) & - & $(t+)$ & - & $(++)$ & - \\
\hline
\end{tabular}

()$=$ inhibition; $(++++)=$ confluent lysis with secondary growth; $(++)$ and $(+++)=$ inhibition with scattered plaques; $++++=$ confluent lysis; $-=$ no lysis.

\section{Nature of the change in phage sensitivity of $80 / 81$ strains}

After lysogenization with the 'converting' phages, strains which originally typed as $\mathbf{8 0 / 8 1}$ became indistinguishable from the strains used as sources of the 'converting' phages. Two changes were involved: immunity to phage $47 \mathrm{C}$; change from 'inhibition' with phages 52 and $52 \mathrm{~A}$ to full susceptibility to attack by these phages. No clones lysogenic for these 'converting' phages were found which showed the 52/52 A 'inhibition' pattern and it thus appeared that the phage sensitivity was closely linked with the presence of the $\mathbf{A}$ prophages.

Several hypotheses may be advanced to explain the mechanism of this change. The change is not one from complete resistance to full sensitivity but from 'inhibition' to full sensitivity. The 'converted' cocci behave as if some partial block in their ability to synthesize phages 52 and $52 \mathrm{~A}$ has been removed. One hypothesis would accord a direct effect of the prophage on the behaviour of the lysogenized cocci. The mechanism of this change might be prophage substitution such as Gorrill (1957) found with the $F$ phage 76, or the prophage might produce an alteration in the surface of cocci harbouring it so that they adsorbed the 52 and $52 \mathrm{~A}$ phages more rapidly. An indirect effect is also possible in which the 'converting' phages act as the selecting agents for phage-sensitive mutants arising spontaneously in $80 / 81$ cultures. If these phage-sensitive mutants were also the only cocci in $80 / 81$ cultures capable of giving a lysogenic response when infected with the 'converting' 
phages, the observed result would be lysogenic cocci fully sensitive to phages 52 and $52 \mathrm{~A}$, but the mechanism of the change would be an indirect one. These hypotheses were examined experimentally.

\section{Lysogenicity of $80 / 81$ strains}

PS 80 was lysogenic and its phage was detectable on strain Bundaberg. Attempts to propagate this phage, $\operatorname{PS} 80 / \mathbf{B}^{\prime} \mathrm{b}$, met with considerable difficulties. It produced only minute fuzzy plaques and confluent lysis was never obtained on agar plates. Preparations from agar had titres of $10^{5} / \mathrm{ml}$. and were heavily contaminated with the lysogenic phage of strain Bundaberg. Attempts to produce high titres of PS 80 by u.v. irradiation were equally unsuccessful. The prophage appeared to be non-inducible but, on one occasion, turbidity readings suggested that lysis of $c .10 \%$ of the cocci had occurred and a preparation was obtained with a plaque count on strain Bundaberg of $3 \times 10^{7} / \mathrm{ml}$. This preparation was used to determine the host range and serology of the phage. This phage lysed only strain Bundaberg; it had no effect on any of the other strains containing 'converting' phages or on any of the propagating strains of the typing phages. Serologically, it was F.

The other $80 / 81$ strains used for 'conversion' to $52 / 52 \mathrm{~A} / 80 / 81$ were examined for the presence of this lysogenic $F$ phage. It was found in strains 6351 and 6402, producing minute plaques on strain Bundaberg. It could not be demonstrated in broth cultures of PS81, 6352 or 9183. No other lysogenic phages were found in any of these $80 / 81$ strains, although they were tested with all the propagating strains as indicators.

All the 'converted' cultures were tested for sensitivity to the $\mathbf{F}$ phage on the assumption that, if prophage substitution had occurred as the result of lysogenization, they should now be sensitive to the phage they had originally carried. All were resistant.

The F phage was sought in a culture of PS80 (308). Prophage 308 is inducible by u.v. irradiation. After irradiation, a lysate containing $2.5 \times 10^{9}$ particles producing plaques on $\mathrm{PS} 80 / \mathrm{ml}$. was obtained which gave a plaque count on strain Bundaberg of $1.5 \times 10^{2} / \mathrm{ml}$. It was concluded that the $\mathrm{F}$ prophage was still present in PS80 cocci lysogenized with phage 308, although induction of prophage 308 in PS80 cocci had almost completely suppressed production of the non-inducible $\mathbf{F}$ phage. Taken as a whole, the results indicated that prophage substitution was not the mechanism of the change in phage sensitivity following lysogenization with the 'converting' phages.

\section{Adsorption of phages 52 and $\mathbf{5 2} \mathbf{A}$}

If lysogenization with the 'converting' phage was correlated with a change in the phage receptors of the surface of the cocci, changes in the adsorption rates of phages 52 and $52 \mathrm{~A}$ should be found. Adsorption experiments were set up, using these two phages mixed with cocci of PS 80, cocci of PS80 (B'b) and PS 80 (684) and, as controls, cocci of PS52 and PS52A. After growth for $4 \mathrm{hr}$. at $37^{\circ}$ in glucose veal broth, volumes of $0.9 \mathrm{ml}$. of this culture were 
placed in tubes to which were added $\mathrm{CaCl}_{2}$ to a final concentration of $300 \mu \mathrm{g}$./ $\mathrm{ml}$. and $0.1 \mathrm{ml}$. of phage suspension diluted to give 80-100 plaques when $0.01 \mathrm{ml}$. volumes of a broth control without cocci were plated. After 10 or $15 \mathrm{~min}$. incubation in a water bath at $37^{\circ}$, the cocci + phage mixtures were centrifuged, the supernatant fluids removed and their content of unadsorbed phage determined by plating on PS52 or PS52A. The sedimented cocci were resuspended in $1 \mathrm{ml}$. volumes of broth and plated on PS52 or 52 A before the end of the latent period, in order to determine the proportion of infected cocci capable of producing phage 52 or phage $52 \mathrm{~A}$.

Table 7. Adsorption and recovery of phage 52 from PS52 cocci, PS 80 cocci and lysogenized PS 80 cocci

\begin{tabular}{|c|c|c|c|c|c|}
\hline \multirow[b]{2}{*}{ Cocci } & \multicolumn{3}{|c|}{ Adsorption for $15 \mathrm{~min}$. at $37^{\circ}$. } & \multirow[b]{2}{*}{$\begin{array}{l}\text { Phage } \\
\text { recovered } \\
\text { in cocci }\end{array}$} & \multirow[b]{2}{*}{$\begin{array}{c}\% \text { of adsorbed } \\
\text { phage } \\
\text { recovered }\end{array}$} \\
\hline & $\begin{array}{l}\text { Phage } \\
\text { added } \\
(a)\end{array}$ & $\begin{array}{c}\text { Phage in } \\
\text { supernatant } \\
\text { (b) }\end{array}$ & $\begin{array}{c}\text { Phage } \\
\text { adsorbed } \\
(a-b)\end{array}$ & & \\
\hline PS52 & 82 & 2 & 80 & 53 & 66 \\
\hline PS 80 & 82 & 1 & 81 & 2 & $2 \cdot 5$ \\
\hline PS $80\left(B^{\prime} b\right)$ & 82 & I & 81 & 37 & $45 \cdot 7$ \\
\hline PS 80 (684) & 82 & 1 & 81 & 30 & 37 \\
\hline
\end{tabular}

Table 7 shows that there was no difference detected in the rate of adsorption of phage 52 to the four kinds of cocci tested, almost complete adsorption having occurred in $15 \mathrm{~min}$. Recovery of phage $\mathbf{5 2}$ from its homologous cocci was not conspicuously efficient, $34 \%$ of the adsorbed phage being lost. From cocci of PS 80, only $2.5 \%$ of the adsorbed phage was recovered while the lysogenic 'converted' cocci yielded $\mathbf{4 5 \cdot 7}$ and $37 \%$ of the adsorbed phage. Similar results were obtained with phage $52 \mathrm{~A}, 96-99 \%$ adsorption occurring in $10 \mathrm{~min}$.

It was concluded that lysogenization with the 'converting' phages was not associated with any alteration in the phage receptors on the surface of the cocci. Inability of the adsorbed phage to penetrate partially-resistant cocci was not excluded by these experiments. However, unpublished observations with another phage-staphylococcus system have shown that a large proportion of the cocci in 'inhibited' cultures are killed, indicating that the block in phage synthesis occurs after phage penetration of these partially resistant organisms.

\section{Spontaneous change from 80/81 to 52/52 A/80/81 typing pattern}

The removal of the block in the ability to synthesize phages 52 and $52 \mathrm{~A}$ might be due to a spontaneous mutation occurring independently of the presence of the 'converting' prophages. The occurrence of phage-sensitive mutants in cultures of PS80 was investigated by a replica plating technique (Lederberg \& Lederberg, 1952). Master plates were inoculated by spreading over their surfaces $0.01 \mathrm{ml}$. of a dilution of broth culture estimated to give 100-200 colonies. After incubation for $24 \mathrm{hr}$. at $37^{\circ}$, the plates were printed on to velvet which was used to inoculate two plates of the soft agar used for phage work; the first of these plates served as a control and the second had been spread with $0.1 \mathrm{ml}$. of a high-titre filtrate of phage 52. These plates were 
incubated at $30^{\circ}$. A trial with PS80 $\left(\mathbf{B}^{\prime} b\right)$ cocci showed that all phagesensitive clones could be recognized either by their small size on the phagetreated plates or by their absence from these plates. By this method, clones of PS 80 fully sensitive to phages 52 and $52 \mathrm{~A}$ were isolated. The site of the phage-sensitive colony having been ascertained by placing the phage-treated plate on top of the replicate without phage, the colony was picked from the plate without phage, grown in broth and tested for its phage reactions.

In four separate experiments (Table 8) 5 mutant colonies were detected among a total of 4375 colonies. Cultures made from these colonies typed as $52 / 52 \mathrm{~A} / 80 / 81$ at the routine test dilution. Two were partially sensitive to phage $47 \mathrm{C}$ and three were resistant. In two experiments, no phage-sensitive colonies were detected among 3082 colonies. The master plates used for replication in these experiments were inoculated with cocci from 4 to $5 \mathrm{hr}$. broth cultures, while for those from which the phage-sensitive mutants were isolated $48 \mathrm{hr}$. cultures were used. This suggests that the organisms of PS80 have a fairly low mutation rate to phage sensitivity and that the mutants are more likely to be detected in older cultures where they have had the opportunity to accumulate. In one experiment, the back mutation to phage 52 'inhibition' was looked for in a culture made from one of these phage-sensitive clones but no partly resistant colonies were found among 1810 sensitives.

Table 8. Occurrence in PS80 cultures of mutant clones sensitive to phages 52 and $52 \mathrm{~A}$

$\begin{array}{cccc}\text { Expt. no. } & \text { No. of plates } & \begin{array}{c}\text { Total no. } \\ \text { colonies }\end{array} & \begin{array}{c}\text { No. of mutant } \\ \text { colonies }\end{array} \\ 1 & 6 & 430 & 2 \\ 2 & 5 & 1016 & - \\ 3 & 8 & 1966 & - \\ 4 & 9 & 963 & 8\end{array}$

Taken as a whole, the results suggest that the 'conversion' to phage sensitivity in strains of 80/81 pattern may be the result of the selection of spontaneous mutants by the 'converting' phages. This hypothesis is supported by the fact that there is no apparent specificity among the lysogenizing phages, 'converted' lysogenic clones being found following the action of the $F$ phage 863 as well as the various A phages. Demonstration of differences in the frequency of lysogenic responses in the original 80/81 cocci as compared with the mutant phage-sensitive cocci would be further evidence in favour of this hypothesis; such evidence is not yet available.

\section{Changes in typing pattern in vivo}

The demonstration that changes in phage typing pattern can occur in vitro either by spontaneous mutation or as a result of artificial lysogenization raises the question of whether such changes also occur in vivo. There seems no a priori reason why they should not occur, but their actual demonstration presents some difficulties. For example, when cultures typing as 80/81 and as 
$52 / 52 \mathrm{~A} / 80 / 81$ are isolated from the same lesion it is impossible to exclude infection with two separate strains.

Examples of changes possibly due to spontaneous mutation have been found in our material. In one case of staphylococcal septicaemia, repeated blood cultures typed as $80 / 81$ at the routine test dilution but on one occasion, a culture which was typed as $52 / 80 / 81$ was isolated. Six single colonies of this culture were picked and typed; five were found to be 52/80/81 and the sixth $80 / 81$.

A pair of cultures was received from Miss K. Key of the Commonwealth Serum Laboratories where they had been used in studies of staphylococcal pathogenicity in mice. The original strain 1406/1, typed as $80 / 81$ with 47 C/52/52 A 'inhibition', while a subculture 1406/2 used in mouse challenge was $47 \mathrm{C} / 52 / 52 \mathrm{~A} / 80 / 81$. The origin of this fully sensitive strain was not known exactly, although it was presumed to have been derived from 1406/1. Neither strain was lysogenic.

One example was found of what appears to be change in typing pattern due to lysogenization. Two cultures were isolated from a patient, on successive days. The first strain (590) typed as 80/81 and was grown from an empyema. The second strain (594) was grown from sputum; it typed as 52/52 A/80/81 and showed numerous phage plaques on the typing plates. From strain 594, two distinct phages were isolated, both serologically $\mathbf{A}$, but differing in host range. Both these phages lysogenized strain 590 cocci and PS 80 cocci, these cultures then typing as either $52 / 52 \mathrm{~A} / 80 / 81$ or $47 \mathrm{C} / 52 / 52 \mathrm{~A} / 80 / 81$, depending on the phage used for lysogenization. The behaviour of these two phages will be discussed in detail elsewhere. The significance of the observation in the present connexion is that it affords suggestive evidence that the patient was originally infected with an 80/81 strain which later encountered a 'converting' phage in the respiratory tract.

\section{DISCUSSION}

Workers concerned with staphylococcal phage typing have generally assumed that some at least of the differences between strains belonging to the same phage group but which display different phage patterns are due to the presence in these strains of prophages which confer immunity to homologous and related phages. Lowbury \& Hood (1953) lysogenized with homologous typingphages thirteen of the strains used to propagate the typing phages and so produced strains immune to one or many phages depending on their original sensitivity. Examination of the data in their paper shows that resistance to a number of the phages occurred in blocks. Thus, lysogenization of PS 47 with phage 47 produced a strain immune to phages $47 \mathrm{~B}$ and $47 \mathrm{C}$ as well as phage 47; lysogenization of PS 7 with phage 7 gave a strain immune to phages 7 , $47,47 \mathrm{C}, 47 \mathrm{D}, 75,75 \mathrm{~A}$ and 76 . With the exception of phage 76 which is serologically F, all these phages are A. Similarly, lysogenization with B phages was followed by immunity to homologous and heterologous $B$ phages.

Rountree (1956) pointed out that immunity following artificial lysogenization was serologically specific, in that lysogenization with an A phage conferred 
immunity to other $\mathbf{A}$ phages but not to $\mathbf{B}$ phages and vice versa. In the present paper, an example is given of this serological specificity of prophage immunity, but it is also shown that this is not a universal phenomenon since the prophage of an $\mathbf{F}$ phage immunized against an $\mathbf{A}$ phage. The reverse case of an $A$ prophage immunizing against the $F$ phage 76 is also implicit in Lowbury \& Hood's data.

Of particular interest was the finding that, following lysogenization with certain $\mathbf{A}$ phages and an $\mathbf{F}$ phage, strains in which the multiplication of the $\mathbf{B}$ phages had been partially blocked became fully sensitive to these $\mathbf{B}$ phages. In these strains, the operational effect of lysogenization was not immunity but sensitivity. However, closer analysis of the phenomenon suggests that the mechanism of the change may be a subtle one, possibly involving selection of phage sensitive mutants able to give a lysogenic response to the 'converting' phages.

On the basis of the present results, it may be postulated that the basic pattern of phage sensitivity in this group of staphylococci is $47 \mathrm{C} / 52 / 52 \mathrm{~A} / 80 / 81$. This may be modified by lysogenization, by spontaneous mutation or by a combination of both mechanisms. Table 9 illustrates the various possibilities,

Table 9. Various phage patterns within the phage group $47 \mathrm{C}, 52$, $52 \mathrm{~A}, 80,81$ and mechanism of their production

$\begin{array}{cc}\begin{array}{c}\text { Coccal } \\ \text { type }\end{array} & \text { Phage pattern at R'TD* } \\ 1 & 47 \mathrm{C} / 52 / 52 \mathrm{~A} / 80 / 81 \\ 2 & 52 / 52 \mathrm{~A} / 80 / 81 \\ 3 & 52 / 52 \mathrm{~A} / 80 / 81 \\ 4 & 80 / 81 \\ & \\ 5 & 47 \mathrm{C} / 52 / 52 \mathrm{~A} / 80 \\ 6 & 52 / 52 \mathrm{~A} / 80 \\ 7 & \mathbf{8 0} \\ 8 & 47 \mathrm{C} / 81 \\ 9 & 81\end{array}$

Mechanism
Basic type; mutation of 4 to phage sensitivity
Mutation of 1 to phage $47 \mathrm{C}$ ' inhibition'
Lysogenization of 1,2 , and 4 , with A prophage
Mutation of 1 to 'inhibition' with phages $47 \mathrm{C}$,
52 and $52 \mathrm{~A}$
Lysogenization of 1 with $\mathrm{F}$ prophage
Lysogenization of 2,3 and 4 with $\mathrm{F}$ prophage
Lysogenization of 4 with $\mathrm{F}$ prophage
Lysogenization of 1 with $\mathrm{B}$ prophage
Lysogenization of 2,3 and 4 with B prophage
routine test dilution.

all of which have been isolated as wild types or produced in the laboratory. Cocci of type 2 can be isolated from cultures of type 1 quite readily and the mutation of type 4 to type 1 cocci has been demonstrated by the replicate plating technique. The mutation of type 1 cocci to type 4 has not been demonstrated in the laboratory but that it may have occurred in nature and been the origin of the 80/81 strains now widely prevalent seems a reasonable hypothesis.

The patterns of prophage immunity produced by lysogenization depends on the original phage sensitivity of the lysogenized cocci. Thus, 80/81 strains lysogenized by the $F$ phage 863 became type 80 , or, when the $F$ phage is 'reduced' in phage-sensitive mutants of $80 / 81$ cocci, $52 / 52 \mathrm{~A} / 80$, while strains of $47 \mathrm{C} / 52 / 52 \mathrm{~A} / 80 / 81$ pattern became $47 \mathrm{C} / 52 / 52 \mathrm{~A} / 80$. These results therefore give some information about the phage and prophage loci in the staphylo- 
cocci. The loci 52, 52 A and 80 appear to be closely linked but not identical. The loci for phage $47 \mathrm{C}$ on the one hand and for phages 81 and 863 on the other are not identical, while those for 81 and 863 are probably identical. It is also apparent that the A 'converting' phages although producing immunity to phage $47 \mathrm{C}$ are not identical with that phage.

This work was supported in part by a grant from the Australian National Health and Medical Research Council.

\section{REFERENCES}

Bynoe, E. T., Elder, R. H. \& Comtors, R. D. (1956). Phage-typing and antibiotic-resistance of staphylococci isolated in a general hospital. Canad. J. Microbiol. 2, 346.

GorriLl, R. H. (1957). Studies on lysogeny in staphylococci. J. gen. Microbiol. 17, 254.

Gorrill, R. H. \& Gray, R. A. (1956). The induction of bacteriophage in staphylococci. J. gen. Microbiol. 14, 167.

Kellaway, C. H., McCallum, P. \& Tebbutt, A. H. (1928). The fatalities at Bundaberg. Report of the Royal Commission. Med. J. Aust. ii, $2,38$.

Lederberg, J. \& Lederberg, E. M. (1952). Replica plating and indirect selection of bacterial mutants. J. Bact. 63, 399.

LowbUry, E. J. L. \& Hood, A. M. (1953). The acquired resistance of Staphylococcus aureus to bacteriophage. J. gen. Microbiol. 9, 524.

Lwoff, A. (1953). Lysogeny. Bact. Rev. 17, 269.

Rountree, P. M. (1949a). The phenomenon of lysogenicity in staphylococci. J. gen. Microbiol. 3, 153.

Rountree, P. M. (1949b). The serological differentiation of staphylococcal bacteriophages. J. gen. Microbiol. 3, 164.

Rountree, P. M. (1952). Serological studies of the multiplication of a staphylococcal bacteriophage. Aust. J. exp. Biol. med. Sci. 30, 567.

Rountree, P. M. (1956). Variations in a related series of staphylococcal bacteriophages. J. gen. Microbiol. 15, 266.

Rountree, P. M. \& Freeman, B. M. (1955). Infections caused by a particular phage type of Staphylococcus aureus. Med. J. Aust. ii, 157.

Sмгтн, H. W. (1948). Investigations on the typing of staphylococci by means of bacteriophage. II. The significance of lysogenic strains in staphylococcal type designation. J. Hyg., Camb. 46, 82.

Williams, R. E. O. \& Rippon, J. E. (1952). Bacteriophage typing of Staphylococcus aureus. J. Hyg., Camb. 50, 320.

Wilson, G. S. \& Atkinson, J. D. (1945). Typing of staphylococci by the bacteriophage method. Lancet, i, 647 . 